\title{
Note: A method for estimating the resolution of nanopositioning systems
}

\author{
Andrew J. Fleming ${ }^{\text {a) }}$ \\ School of Electrical Engineering and Computer Science, University of Newcastle, Callaghan, \\ NSW 2308, Australia
}

(Received 11 October 2011; accepted 13 July 2012; published online 1 August 2012)

This Note describes a new technique for estimating the resolution of nanopositioning systems. By recording the voltage applied to an actuator and performing a filtering operation, the position noise and resolution can be estimated. This technique is simple to apply in practice and does not require any additional sensors or specialized equipment. (c) 2012 American Institute of Physics. [http://dx.doi.org/10.1063/1.4739508]

The most straightforward and conclusive method for measuring the positioning noise of a nanopositioning system is to measure the position noise directly. However, this approach is not often possible as an additional sensor is required with lower noise and a significantly higher bandwidth than the closed-loop system. Instead, the position noise is typically predicted from measurements of the sensor noise. ${ }^{1-4}$ However, this approach tends to underestimate the position noise since the influence of the high-voltage amplifier is neglected.

In this Note, a new method is proposed for measuring the resolution of nanopositioning systems. While the system is operating in closed-loop, the voltage applied to the piezoelectric actuator is recorded, either with a spectrum analyzer or in the time domain. The data is then filtered by a model of the system dynamics or a frequency response to produce an estimate of the positioning noise and resolution. This approach captures the effect of all electronic and sensor noise without the need for additional sensors.

Compared to standard methods, ${ }^{1-4}$ the proposed technique has two significant benefits. First, only a single measurement is required to capture both the amplifier noise and sensor noise. Other techniques require the individual measurement of each noise source. Second, the frequency response or model of the open-loop system is easier to obtain than the closed-loop model, which is required by other methods. Furthermore, in many practical situations, the controller may be manually tuned and not precisely known, which makes it difficult to estimate the closed-loop sensitivity functions.

When a nanopositioner has settled to a commanded location, a small amount of random motion remains due to the sensor noise, amplifier noise, and external disturbances. The residual random motion means that two adjacent commanded locations may actually overlap, which can cause manufacturing faults or imaging artifacts. To avoid these eventualities, it is critical to know the minimum distance between two adjacent but unique locations.

Since the noise sources that contribute to random position errors have a potentially large dispersion, it is impractically conservative to specify a resolution where adjacent regions never overlap. Instead, it is preferable to state the probability that the actual position is within a certain bound. Consider the example of random positioning errors plotted in Figure 1(a). Observe that the peak-to-peak amplitude of random motion is bounded by $\delta_{x}$ and $\delta_{y}$; however, this range is occasionally exceeded. If the random position variation is assumed to be Gaussian distributed, the probability density functions of three adjacent points, spaced by $\delta_{x}$, are plotted in Figure 1(b). In this example, $\delta_{x}$ is equal to $\pm 3 \sigma_{x}$ or $6 \sigma_{x}$, where $\sigma_{x}$ is the standard deviation or rms value, so $99.7 \%$ of the samples fall within the range specified by $\delta_{x}$. Restated, there is a $0.3 \%$ chance that the position is exceeding $\delta_{x}$ and

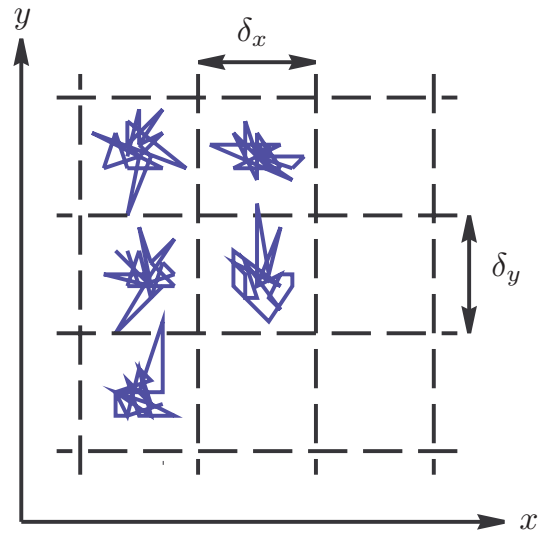

(a) Two dimensional random motion

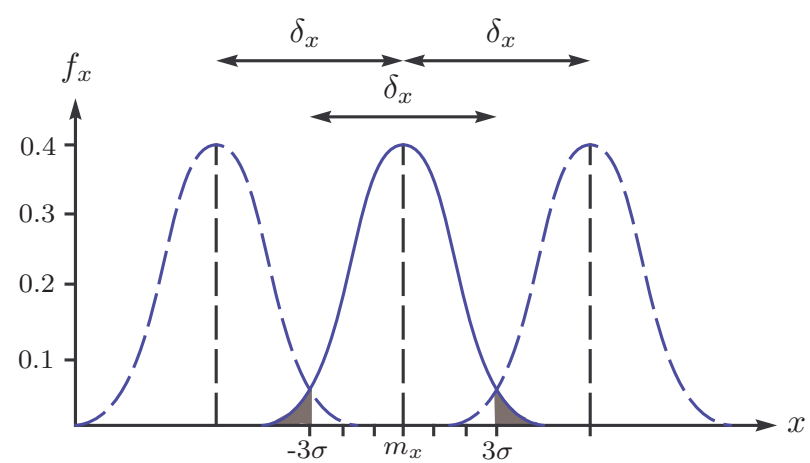

(b) The probability density functions of three adjacent points on the $x$-axis

FIG. 1. The random motion of a two-dimensional nanopositioner.

a) Andrew.Fleming@ Newcastle.edu.au. 


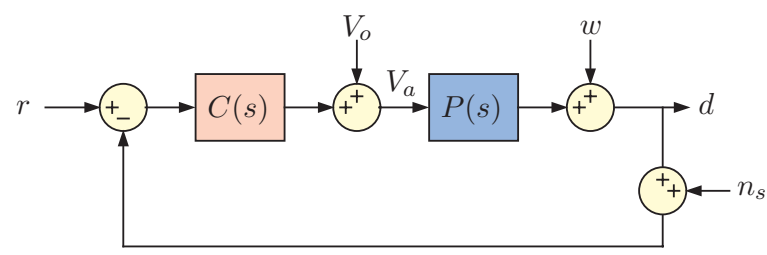

FIG. 2. A single axis feedback control loop with a plant $P(s)$ and controller $C(s)$.

straying into a neighboring area, this probability is shaded in grey.

In nanopositioning applications, a $99.7 \%$ probability that the position falls within $6 \sigma_{x}$ is an appropriate definition for the resolution. To be precise, this definition should be referred to as the $6 \sigma$-resolution and specifies the minimum spacing between two adjacent points that do not overlap $99.7 \%$ of the time. If the noise is non-Gaussian, the 99.7 percentile, or peak-to-peak value, must be measured directly rather than predicted from the rms value.

In other applications where more or less overlap between points is tolerable, another definition of resolution may be more appropriate. For example, the $4 \sigma$ resolution would result in an overlap $4.5 \%$ of the time, while the $10 \sigma$ resolution would almost eliminate the probability of an overlap. Thus, it is not the exact definition that is important; rather, it is the necessity of quoting the resolution together with its statistical definition.

The foremost sources of noise in a nanopositioning application are the amplifier noise, sensor noise, and external disturbances. As shown in Figure 2, the amplifier noise $V_{o}$ appears at the plant input. In contrast, the external disturbances $w$ act at the plant output and the sensor noise $n_{s}$ disturbs the measurement. For the sake of simplicity, the voltage amplifier is considered to be part of the controller.

Considering the limitations of standard noise prediction techniques discussed in the introduction, there is a need for a practical procedure that can accurately estimate the closedloop resolution of a nanopositioning system. A new procedure that fulfills this goal is described in the following. The applied-voltage method predicts the positioning noise from a measurement of the closed-loop voltage applied to the nanopositioner. The recorded voltage is then filtered by the open-loop frequency response or model of the plant to reveal the closed-loop resolution.

The feedback diagram of a single-axis control loop is illustrated in Figure 2. The output position $d$ is equal to the applied voltage $V_{a}$ filtered by the plant $P(s)$. Hence, the position noise due to the amplifier and sensor can be estimated by measuring the closed-loop voltage noise $V_{a}$ and filtering it by the plant dynamics. This measurement can be performed in the time or frequency domain and does not require any additional sensors.

If the power spectral density of $V_{a}$ is recorded, the spectral density of the position noise $d$ is

$$
S_{d}(f)=S_{V_{a}}(f)|P(j 2 \pi f)|^{2},
$$

where $S_{d}(f)$ is the power spectral density of $d, S_{V_{a}}(f)$ is the power spectral density of $V_{a}$, and $P(j 2 \pi f)$ is the frequency response of the system or model. The rms value of $d$ can then be computed from the Wiener Khinchin relations

$$
\sigma_{d}=\sqrt{\int_{0}^{\infty} S_{d}(f) d f} .
$$

Assuming a Gaussian distribution, the $6 \sigma$-resolution is then $\delta$ $=6 \times \sigma_{d}$. In the time domain, the position noise estimate is $V_{a}$ filtered by the plant model $P(s)$, that is, $d(t)=P(s) V_{a}(t)$.

To ensure a valid estimate of resolution, there are a number of practical considerations that should be considered. As the output voltage noise of a high-voltage amplifier is typically in the range of 1 to $20 \mathrm{mVp}$-p, the signal must be preamplified. In addition, the potentially large (hundreds of volts) $\mathrm{dc}$ offset must be removed by ac coupling. In nanopositioning applications, frequencies down to $0.1 \mathrm{~Hz}$ are of interest so the cut-off frequency of the high-pass filter must be below $0.1 \mathrm{~Hz}$. For example, the Stanford Research SR560 amplifier has a high-pass cut-off frequency of $0.03 \mathrm{~Hz}$.

When utilizing low-frequency ac coupling, it is important to allow the transient response of the filter to decay before recording data. When measuring small ac signals with large dc components, it may take in excess of 20 time-constants for the transient response to become negligible. With an ac coupling frequency of $0.03 \mathrm{~Hz}$, the required delay is approximately $100 \mathrm{~s}$. More generally, the measurement delay $T_{D}$ should be at least $T_{D}=\frac{20}{2 \pi f_{c}}$, where $f_{c}$ is the high-pass filter cut-off.

If frequency domain data is recorded, the measured spectrum should be split into two or three decades to provide sufficient resolution and range. For example: 0 to $12 \mathrm{~Hz}$, $12 \mathrm{~Hz}$ to $1.2 \mathrm{kHz}$, and $1.2 \mathrm{kHz}$ to $12 \mathrm{kHz}$. The data should have a frequency range of at least five times the amplifier bandwidth. The rms value and $6 \sigma$-resolution can then be found by evaluating the integral in Eq. (2). Alternatively, it may be more convenient to use the spectral density $\sqrt{S_{V_{a}}(f)}$ with units of $V / \sqrt{\mathrm{Hz}}$. In this case, the standard deviation is

$$
\sigma=\int_{0}^{\infty} \sqrt{S_{V_{a}}(f)}|P(j 2 \pi f)| d f .
$$

Time domain recordings require a choice of the recording length and sampling rate. The length of each recording is defined by the lowest spectral component under consideration. With a lower frequency limit of $0.1 \mathrm{~Hz}$, a record length of at least ten times the minimum period is required to obtain a statistically meaningful estimate of the rms value, which implies a minimum recording length of at least $100 \mathrm{~s}$. A longer record length is preferable, but may not be practical.

When selecting the sampling rate, the highest significant frequency that influences position noise should be considered. Since the sensor noise is low-pass filtered by the closed-loop response of the control loop, the highest significant frequency is usually the bandwidth of the voltage amplifier. An appropriate choice of sampling rate is 15 times the amplifier bandwidth. This allows a non-ideal anti-aliasing filter to be utilized with a cut-off frequency of five times the amplifier bandwidth. Since the noise power of a first-order amplifier drops to $3.8 \%$ at five times the bandwidth, this technique captures the 
TABLE I. Recommended parameters for time domain noise recordings.

Amplifier bandwidth

Anti-aliasing filter cut-off frequency

Sampling rate

Record length

majority of noise power. The recommended parameters for time-domain noise recordings are summarized in Table I.

In the following, the applied-voltage technique is employed to estimate the resolution of the piezoelectric tube described previously. ${ }^{5}$ The tube displacement sensitivity is $171 \mathrm{~nm} / \mathrm{V}$ which implies a range of approximately $68 \mu \mathrm{m}$ with a $\pm 200 \mathrm{~V}$ excitation. The voltage amplifier used to drive the tube is a Nanonis HVA4 high-voltage amplifier with a gain of 40. The position sensor is an ADE Tech 4810 Gaging Module with 2804 capacitive sensor. This sensor has a full range of $\pm 100 \mu \mathrm{m}$ and a sensitivity of $0.1 \mathrm{~V} / \mu \mathrm{m}$.

A model was identified from the frequency response using the MATLAB function invfreqs. The resulting parameters are

$$
P(s)=\frac{B(s)}{A(s)}=\frac{0.01151 s^{2}+116 s+2.541 \times 10^{6}}{s^{2}+66.73 s+2.658 \times 10^{7}} \mu \mathrm{m} / \mathrm{V} .
$$

Alternatively, the model could be obtained from time-domain recordings using a white-noise or step response excitation. ${ }^{6,7}$

For the sake of demonstration, an analog integral controller was implemented with a closed-loop bandwidth of $10 \mathrm{~Hz}$. After setting the reference input to zero, the voltage applied to the nanopositioner was preamplified by an SR560 amplifier with a gain of 500 and an ac coupling frequency of $0.03 \mathrm{~Hz}$. This signal was recorded for $100 \mathrm{~s}$ with a sampling rate of $30 \mathrm{kHz}$.

To estimate the closed-loop positioning noise, the noise recording was filtered by the model (4) using the MATLAB function $1 \mathrm{sim}$. The distribution of the resulting displacement estimate, plotted in Figure 3(a), has a rms value of $0.24 \mathrm{~nm}$ and a $6 \sigma$ resolution of $1.4 \mathrm{~nm}$. Since $1.4 \mathrm{~nm}$ is greater than $6 \times 0.24 \mathrm{~nm}$, the distribution is slightly more dispersed than a Gaussian distribution.

In Figure 3(b), the estimated closed-loop noise is used to visualize the expected two-axis resolution. This figure is

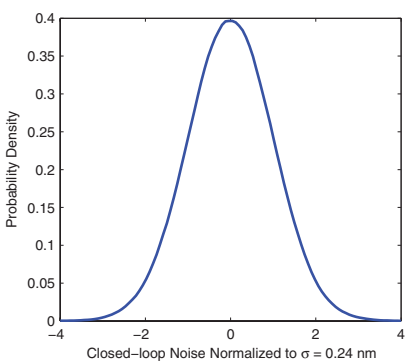

(a) Distribution of the predicted noise

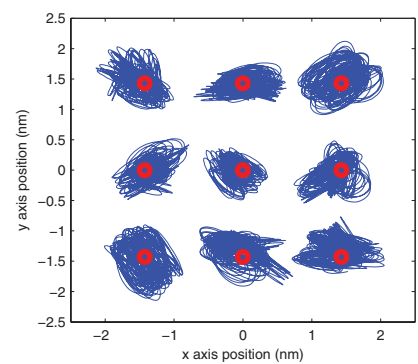

(b) Simulated constellation diagram of nine two-dimensional points spaced by the $6 \sigma$ two-dimen
FIG. 3. (a) The distribution of position noise in a piezoelectric tube nanopositioner with a closed-loop bandwidth of $10 \mathrm{~Hz}$. (b) The simulated position noise is plotted in two dimensions to illustrate the concept of resolution is two axes.

only a simulation but is useful for illustrating the concept of $6 \sigma$ resolution in two axes. Nine $100 \mathrm{~ms}$ data sets were taken randomly from the estimated position noise and plotted on a constellation diagram with a spacing equal to the prescribed resolution.

The resolution obtained above can also be compared to that predicted from measurements of the open-loop sensor and amplifier noise. ${ }^{8}$ With the same closed-loop bandwidth $(10 \mathrm{~Hz})$ the standard frequency domain approach estimates the closed-loop resolution to be $1.5 \mathrm{~nm}$, which compares well to the $1.4 \mathrm{~nm}$ predicted by the applied-voltage technique.

${ }^{1}$ A. Sebastian, A. Pantazi, H. Pozidis, and E. Elefthriou, IEEE Control Syst. 28(4), 26-35 (2008).

${ }^{2}$ S. S. Aphale, B. Bhikkaji, and S. O. R. Moheimani, IEEE Trans. Nanotechnol. 7(1), 79-90 (2008).

${ }^{3}$ A. J. Fleming, IEEE Trans. Mechatron. 15(3), 433-447 (2010).

${ }^{4}$ A. J. Fleming, S. S. Aphale, and S. O. R. Moheimani, IEEE Trans. Nanotechnol. 9(4), 438-448 (2010).

${ }^{5}$ J. Maess, A. J. Fleming, and F. Allgöwer, Rev. Sci. Instrum. 79, 015105 (2008).

${ }^{6}$ L. Ljung, System Identification: Theory for the User (Prentice Hall, Upper Saddle River, NJ, 1999).

${ }^{7}$ B. Ninness and A. Wills, Proceedings of the IFAC Symposium on System Identification (IFAC, Laxenburg, Austria, 2006), pp. 301-307.

${ }^{8} \mathrm{~A}$. J. Fleming, in Proceedings of the IEEE International Conference on Robotics and Automation, St. Paul, MN (IEEE, NJ, USA, 2012), pp. 4786-4791. 